\title{
Multilayered Pipe Cutting Test for Remote Handling Maintenance
}

\author{
Haibin Chen, ${ }^{1}$ Jianwen Guo, ${ }^{1}$ Zhenzhong Sun, ${ }^{1}$ Xuejun Jia, ${ }^{2}$ and Hong Tang ${ }^{2}$ \\ ${ }^{1}$ School of Mechanical Engineering, Dongguan University of Technology, Dongguan 523808, China \\ ${ }^{2}$ Dongguan Neutron Science Center, Dongguan 523890, China \\ Correspondence should be addressed to Zhenzhong Sun; sunzhzh@tom.com
}

Received 10 March 2015; Revised 17 May 2015; Accepted 21 May 2015

Academic Editor: Arkady Serikov

Copyright (c) 2015 Haibin Chen et al. This is an open access article distributed under the Creative Commons Attribution License, which permits unrestricted use, distribution, and reproduction in any medium, provided the original work is properly cited.

\begin{abstract}
Based on the requirements for remote handling maintenance (RHM) of China Spallation Neutron Source (CSNS) multilayered pipes, pipes cutting tests were performed under remote handling maintenance conditions. In this study, the results were obtained from different cutting directions and supporting intensities of pipe baseplates comparisons: When enough power was provided and the blade gripper did not slip, the cutting direction had little impact on the cutting capacity but more on the fault surface; the clearance between the blades caused the rotating torque; for remote handling maintenance, good horizontal support of the longhandled lever of the hydraulic cutter was required. Significant conclusions were made for multilayered pipe cutting that are crucial for auxiliary tools development for remote handling maintenance.
\end{abstract}

\section{Introduction}

Nuclear devices are associated with the research and application of high-energy physics, and typical nuclear devices globally include the Large Hadron Collider (LHC) developed by the European Organization for Nuclear Research (CERN) [1], the Spallation Neutron Source (SNS) developed by the U.S. Oak Ridge National Laboratory (ORNL) [2], the International Thermonuclear Experimental Reactor (ITER) [3], the Beijing Electron Positron Collider (BEPC) [4], and the China Spallation Neutron Source (CSNS) which is under construction [5]. These devices provide the basic conditions for the realization of strategic, basic, and forward-looking science and technology research.

Remote handling maintenance (RHM) [6] is the act of monitoring and maintaining equipment in a safety zone away from the site by applying a manipulator (robot) or special equipment inside the nuclear device to reduce the exposure dose and labor intensity for personnel. Presently, RHM technology is an important and indispensable tool for maintaining radioactive parts for large-scale scientific facilities [7, 8].

Nuclear devices are equipped with a cooling system (generally 5 to $20 \mathrm{~K}$ ) that uses multilayered pipes (such as liquid cooling medium or gas delivery pipes), to reduce the amount of heat produced during particle reactions. Multilayered pipes, as a channel to transfer the cooling medium, are an important infrastructure for nuclear devices [9]. Considering the impact of age and irradiation damage on performance, multilayered pipes must be inspected and replaced regularly. Since a radiation environment is involved, remote technology is required for multilayered pipe maintenance.

Cutting is a necessary step in the RHM of multilayered pipes [10]. A multilayer structure is generally adopted in the multilayered pipes of nuclear devices, which increases the difficulty of pipe cutting and requires higher performance of the cutting tools for RHM while enhancing the pipe performance. The hydraulic cutter will be exposed to a normal pressure and temperature environment in the deepwell helium container, and radiation level will be low at 400 to $1000 \mathrm{mSv}$; that radiation environment has less influence on the hydraulic tool.

Based on the requirements for RHM of CSNS multilayered pipes, multilayered pipe cutting tests were performed with cutting tools remotely deployed. By comparing the results of different supporting intensities of pipe baseplates and different cutting directions, significant conclusions were 


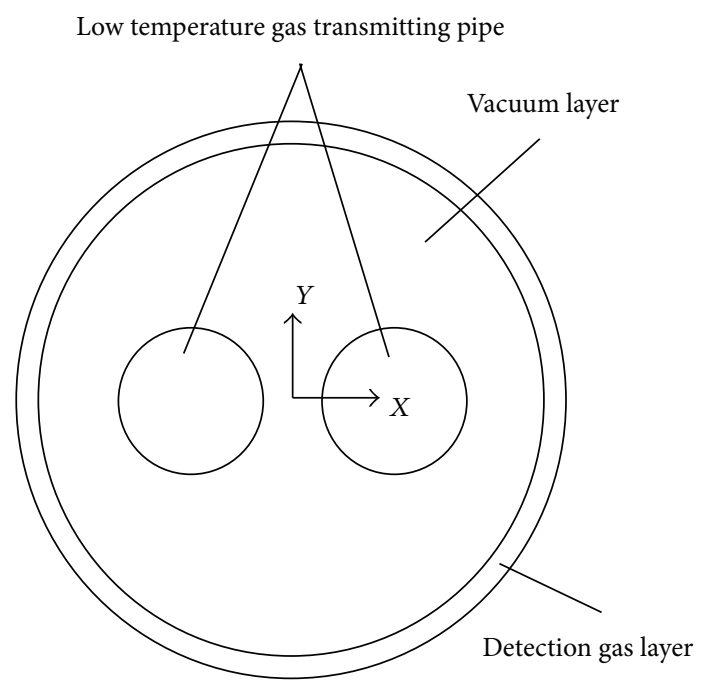

Figure 1: Cross section of the pipe.

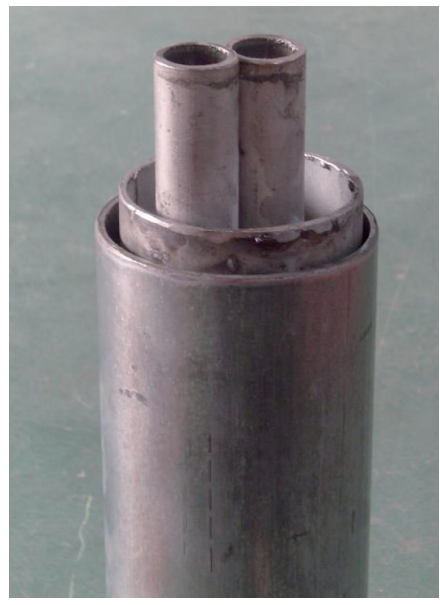

(a) Pipes

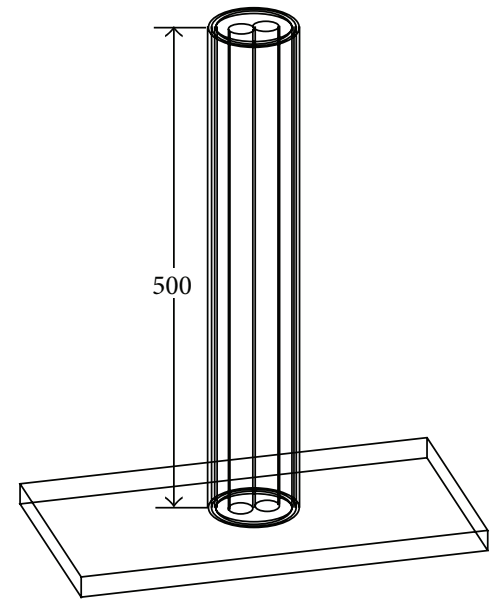

(b) Pipe model

Figure 2: Pipe sample.

obtained for multilayered pipe cutting, which provide a basis for auxiliary tooling development for RHM.

\section{Material and Methods}

CSNS multilayered pipes were made from flexural 316stainless steel multilayered pipe and have a length of 4 to $5 \mathrm{~m}$ per pipe. The cross section of the pipes is shown in Figure 1: the middle part is the cooling medium section (two pipes which are $\phi 25 \mathrm{~mm}$ and $2 \mathrm{~mm}$ thickness), the inner part is the vacuum-heat insulating layer (pipe which is $\phi 80 \mathrm{~mm}$ and $2 \mathrm{~mm}$ thickness), and the outer part is the gas-leakagedetection layer (pipe which is $\phi 100 \mathrm{~mm}$ and $2 \mathrm{~mm}$ thickness). A pipe with the same specifications as that shown in Figure 2 (500 $\mathrm{mm}$ in length) was taken as a sample, and the pipe was welded on a $L 400 \mathrm{~mm} \times W 220 \mathrm{~mm} \times H 20 \mathrm{~mm}$ steel plate.
Pipes are remotely replaced after being cut: Firstly, the hydraulic cutter will be moved away after finishing the cutting. Secondly, a long-handled pneumatic tong will be lifted up in the crane and moved into the deep-well helium container by remote handing. Thirdly, 1 or 2 operating crossbars can be fixed on the long handle. Fourthly, the long-handled pneumatic tong should be located by dropdown pipes according to the operating crossbar. Finally, catch the aim pipes and take them away from deep-well helium container. New pipes are assembled and hanged into the deep-well helium container after being cleaned up.

The hydraulic cutter shown in Figure 3(a) and the hydraulic pump with $3675 \mathrm{~W}$ of power and blades as shown in Figure 3(b) were used. Because of the heavy weight of the hydraulic cutter and the fuel feed pipe, long handle was hoisted and controlled with a long-handled lever (Figure 4). 


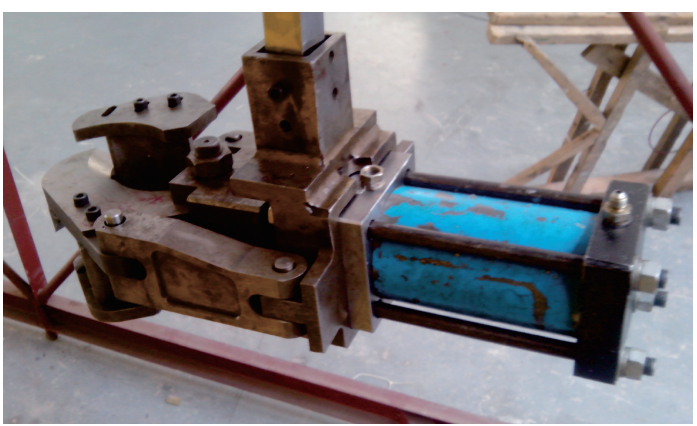

(a) Cutter

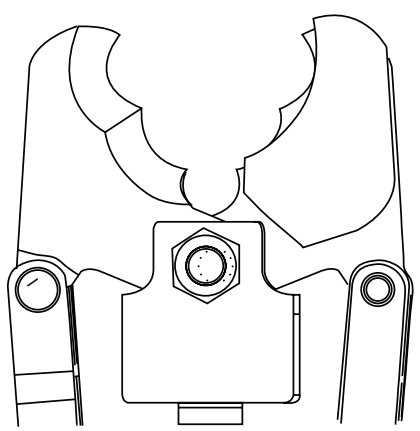

(b) Blade

FIGURE 3: Hydraulic cutter.

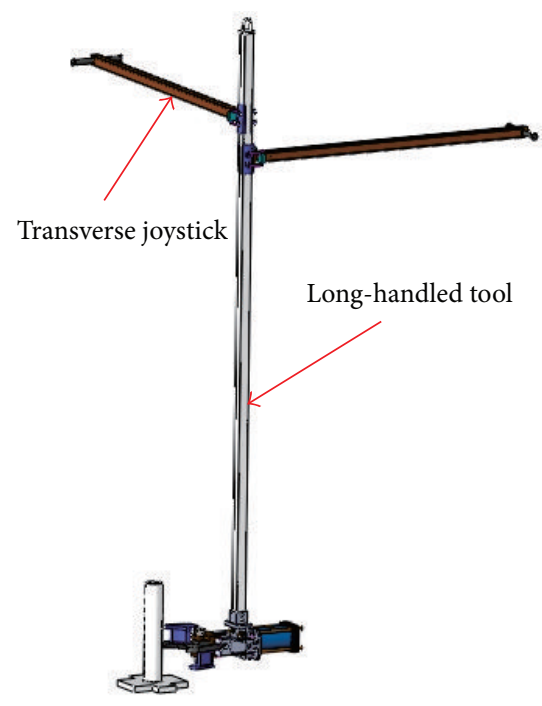

FIgURE 4: Long-handled lever.

\section{Results}

To observe the impact of different cutting directions on the performance, series and parallel cutting were considered.

3.1. Series Cutting. Series cutting refers to a parallel arrangement of the cutting edge and the two midpipes for cutting (Figure 5). During the cutting process, the blade of the hydraulic cutter squeezes the outer pipe and transmits the bite force to the midpipe, causing the midpipe to compress to a flat shape. When the tool nose breaks through the top of the pipe wall because of the lack of die support in the pipe, the shear force deforms the area surrounding the tool nose and collapses in the slit of the pipe top. Then, the cutting wastes are inverted [11] and are ruptured, which forms a fault zone. The fault surface can be divided into the euphotic part, shear part, shear droop, and burr. No relative sliding was found in the two midpipes, and the periphery pipes that were cut completely detached. The blade produced a large reverse torque during the cutting process causing the pipe to tilt. A longer pipe baseplate ( $400 \mathrm{~mm}$ in length) eliminated the torsion, so the cutting process was smooth. The maximum pressure output of the hydraulic pump is shown in a pressure gauge on the hydraulic pump at $70 \mathrm{MPa}$. The fault surface formed during cutting is shown Figure 6.

3.2. Parallel Cutting. Parallel cutting is when the cutting edge is perpendicular to the two midpipes (Figure 7), and it also involves a compressive flat stage. Different from series cutting, the small size of the baseplate $(220 \mathrm{~mm}$ in width) causes the baseplate to tilt, changes the cutting direction, and fails to cut the pipe. The maximum pressure output of the hydraulic pump is shown in a pressure gauge on the hydraulic pump at $74 \mathrm{MPa}$. The fault surface tilt is shown in Figure 8.

To complete the cutting, the testing pipe sample was improved by applying $100 \mathrm{~mm}$ steel plates at both ends of the baseplate width and welding them together (Figure 9). The improved pipe baseplate was used in subsequent testing, and it produced enough support strength to allow the cutting to be completed smooth. After experiencing peripheral pipe flattening, the midpipe was compressed and fractured successively. In parallel cutting, larger power was required, and the fault surface was torn from inside to outside. 

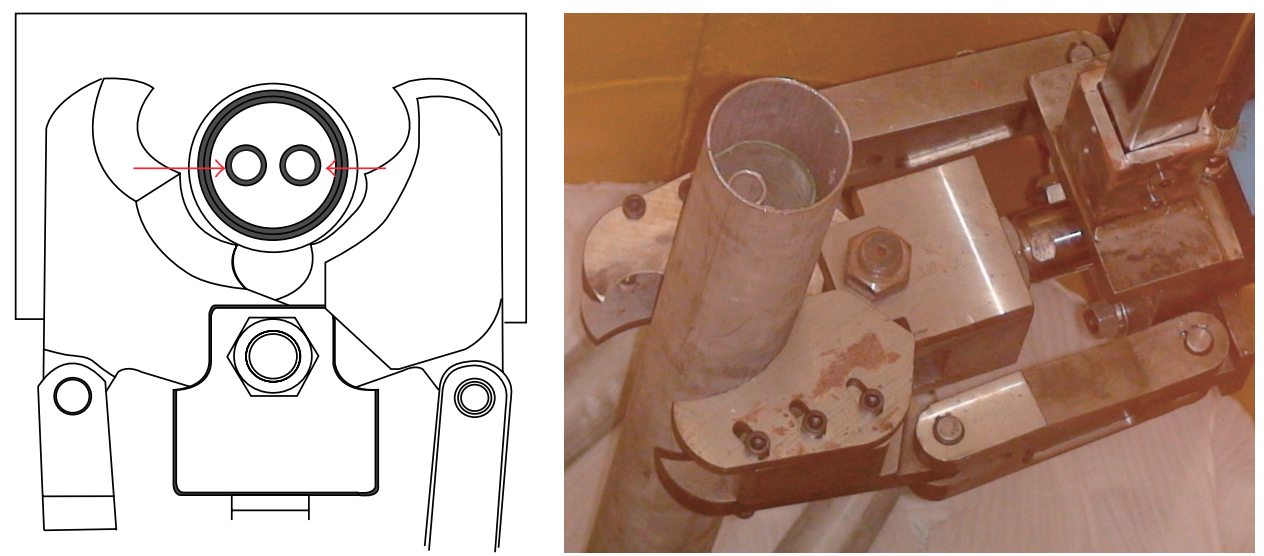

FIGURE 5: Series cutting.

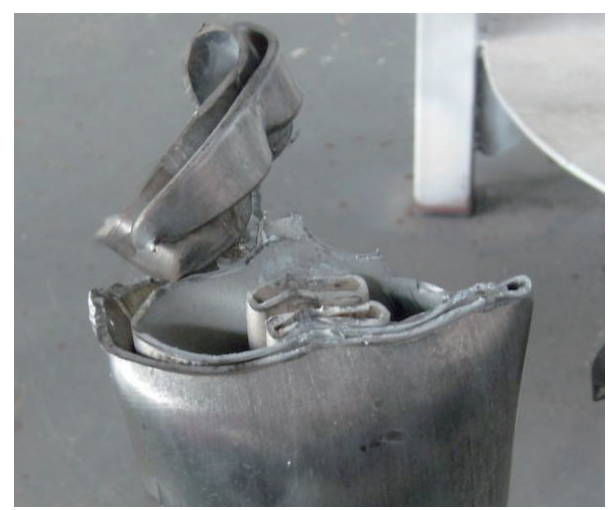

FIgURE 6: Fault surface of series cutting.

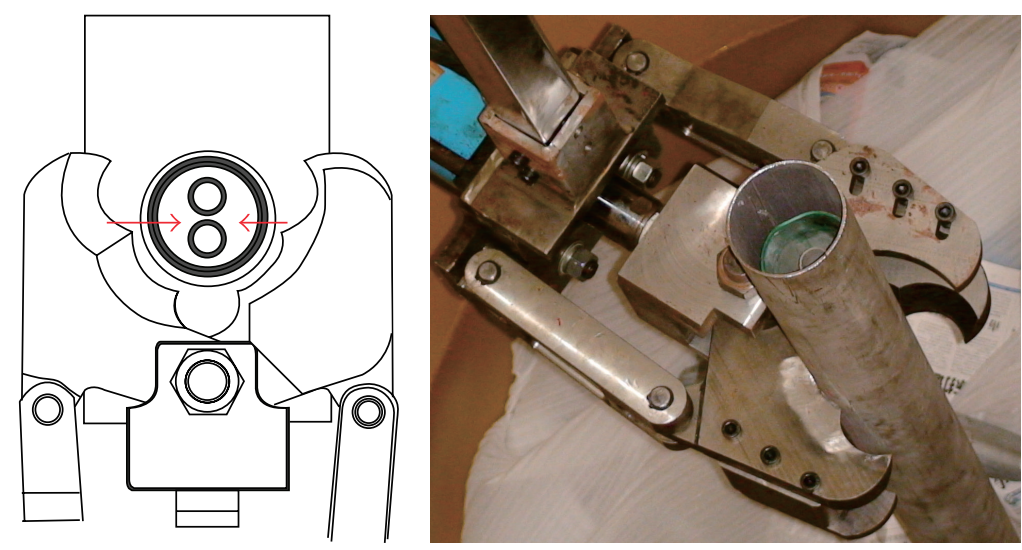

Figure 7: Parallel cutting.

\section{Discussion}

The cutting capacity, fault surface, shear torsion, and long handle tools are analyzed by comparing the cutting direction and pipe support strength.

4.1. Impact of the Cutting Direction. Statistically, $70 \mathrm{MPa}$ pump pressure was required by series cutting, while parallel cutting requires $74 \mathrm{MPa}$, and pressure provided by the hydraulic pump is more than $80 \mathrm{MPa}$. Therefore, power is enough. From the results, both series and parallel cutting successfully cut the pipes. Series cutting required relatively smaller power, indicating that the cutting direction only affected the cutting capacity. The fault surface was clear and smooth, so the fracture was caused by extrusion rather than cutting. According to Figure 1, the pipe wall is $2 \mathrm{~mm}$ thick, and the cutting thickness reached $4 \mathrm{~mm}$ after extrusion, which is quite large for the blade size used. With increasing 


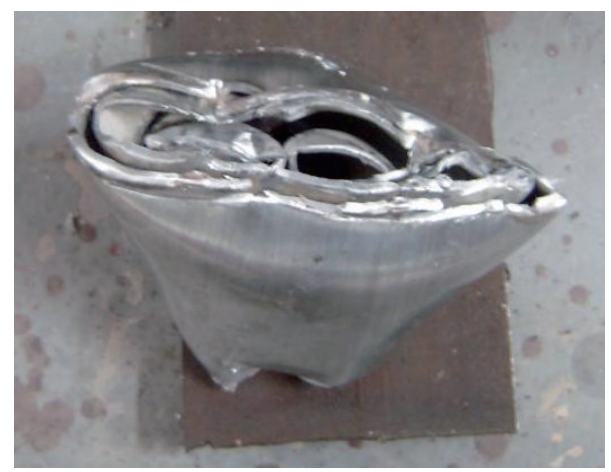

FIgURE 8: Tilt cutting edge.

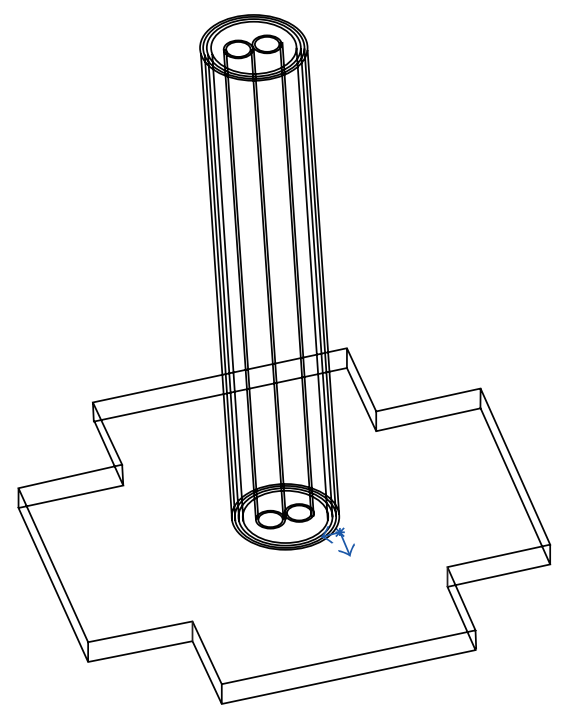

FIGURE 9: Improved pipe baseplate.

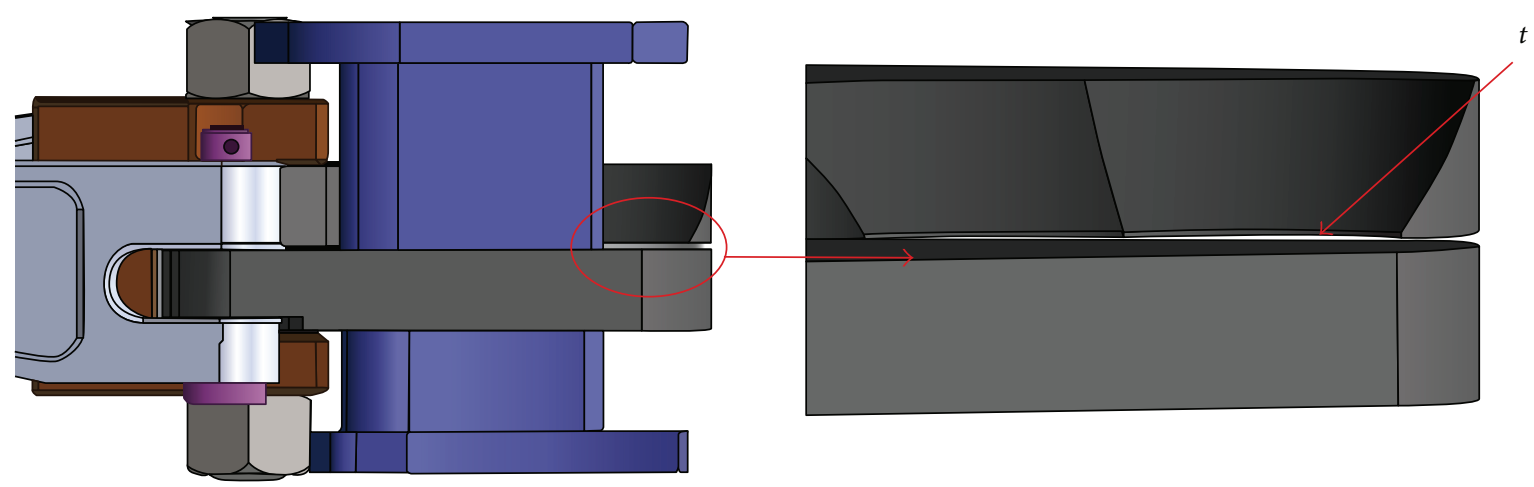

FIgURE 10: Clearance between the two blades.

shear stress, the materials at both ends were first squeezed to fracture rather than cutting. The impact of the cutting direction on the fault surface was limited to the difference in the openings: for series cutting, the unilateral pipe was cut upon squeezing by the blade and the inner pipe was eventually cut after it contacted another pipe; however, for parallel cutting, both ends of the pipe were squeezed by the blade and fractured simultaneously, so the fault surfaces were relatively symmetric.
4.2. Impact of the Supporting Intensities of the Pipe Baseplates. In the parallel cutting test, cutting was affected by the tilt, but it was completed smoothly after improving the baseplate width. Theoretically, the two blades move in a plane, but, in reality, a clearance, $t$, exists between the blades (Figure 10), which causes the cutting to become the origin of the rotating torque. As a result of the huge bite force of the cutter, the amount of force on this clearance can produce greater torque causing the pipe to tilt. To cut the abovementioned pipe 
sample, a force of $74 \mathrm{KN}$ would be required according to calculation. If $t=1 \mathrm{~mm}$, then the rotating torque $M=F * L=$ $74 \mathrm{Nm}$.

According to the two tests, when there was enough supporting intensity provided by the baseplate, the cutter tilted to the left and right, and results similar to that of pipe tilt were obtained. The large shear force was converted to rejection tension between the blades making the clearance bigger, and the pipe extrusion fracture became difficult. Therefore, for RHM and pipe cutting, in addition to maintaining a particular vertical degree, an adequate level of support must be provided by the long-handled lever of the hydraulic cutter to ensure smooth cutting.

\section{Conclusions and Further Works}

Based on the analysis of the experimental results, the following conclusions were obtained:

(1) When enough power was provided by the hydraulic pump and the blade gripper did not slip, the cutting direction had little impact on the cutting capacity.

(2) Comparing Figures 6 and 8, for serial cutting, the impact of the cutting direction on the fault surface was limited to the unilateral fracture upon blade extrusion. However, for parallel cutting, both sides of the pipe were fractured by blade extrusion, and the fault surface was symmetrical.

(3) The clearance, $t$, between the blades caused the cutting to become the origin of the rotating torque. If $t=1 \mathrm{~mm}$, then the rotating torque $M=74 \mathrm{Nm}$.

(4) Horizontal cutting of the shear as well as keeping the pipe at a particular verticality was important. For RHM, good horizontal support of the long-handled lever of the hydraulic cutter was required.

Further works of the research are as follows:

(1) To manufacture a better cutter structure which can limit the clearance, $t$, or design a pipe with limited structure on the cutter.

(2) To design appropriate cutter blades based on bionics which are expected to have better cutting of the multilayered pipes.

(3) An improvement that will be made in blade material for a better coordinate of the hardness and tenacity.

\section{Conflict of Interests}

The authors declare that there is no conflict of interests regarding the publication of this paper.

\section{Acknowledgments}

The study was supported by the National Natural Science Foundation of China (Grant no. 71201026), the Project of the Department of Education of Guangdong Province (nos. 2013KJCX0179, 2014KTSCX184, and 2014KGJHZ014), the Development Program for Excellent Young Teachers in Higher Education Institutions of Guangdong Province (no. Yq2013156), the China Spallation Neutron Source Electromechanical Technology R\&D Joint Laboratory Foundation (no. ZD120512), the Dongguan Social Science and Technology Development Project (no. 2013108101011), and the Science and Technological Program for Dongguan's Higher Education, Science and Research Institutions (no. 2014106101007).

\section{References}

[1] T. S. Virdee, "The LHC project: the accelerator and the experiments," Nuclear Instruments and Methods in Physics Research, Section A: Accelerators, Spectrometers, Detectors and Associated Equipment, vol. 623, no. 1, pp. 1-10, 2010.

[2] G. S. Bauer, "Physics and technology of spallation neutron sources," Nuclear Instruments and Methods in Physics Research, Section A: Accelerators, Spectrometers, Detectors and Associated Equipment, vol. 463, no. 3, pp. 505-543, 2001.

[3] N. Holtkamp, "An overview of the ITER project," Fusion Engineering and Design, vol. 82, no. 5-14, pp. 427-434, 2007.

[4] M. Ye and Z. Zheng, "BEPC, the Beijing electron-positron collider," Nuclear Physics B-Proceedings Supplements, vol. 1, no. 2, pp. 207-216, 1988.

[5] J. Wei, H. Chen, Y. Chen et al., "China spallation neutron source: design, R\&D, and outlook," Nuclear Instruments and Methods in Physics Research. Section A: Accelerators, Spectrometers, Detectors and Associated Equipment, vol. 600, no. 1, pp. 10-13, 2009.

[6] B. Haist, "Setting up and managing a remote maintenance operation for fusion," Fusion Engineering and Design, vol. 83, no. 1012, pp. 1841-1844, 2008.

[7] A. C. Rolfe, P. Brown, P. Carter et al., "Report on the first remote handling operations at JET," Fusion Engineering and Design, vol. 46, no. 2-4, pp. 299-306, 1999.

[8] H. Bernhard, “The remote handling systems for ITER," Fusion Engineering and Design, vol. 86, no. 6-8, pp. 471-477, 2011.

[9] R. Shuff and S. Mills, "A study of pipe jointing technology with reference to ITER requirements," Fusion Engineering and Design, vol. 84, no. 7-11, pp. 1767-1769, 2009.

[10] O. Piñeiro, C. Fernández, M. Medrano et al., "Feasibility study of the cut and weld operations by $\mathrm{RH}$ on the cooling pipes of ITER NB components," Fusion Engineering and Design, vol. 84, no. 7-11, pp. 1515-1520, 2009.

[11] H. Dajun, L. Zhenhua, and H. Ming, "To design blades for shearing tubes and sections," China Metal Forming Equipment \& Manufacturing Technology, vol. 32, no. 5, pp. 17-19, 1997. 


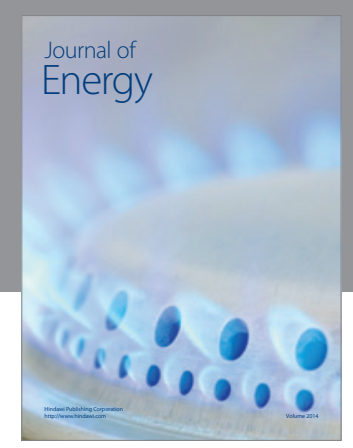

Journal of

Industrial Engineering
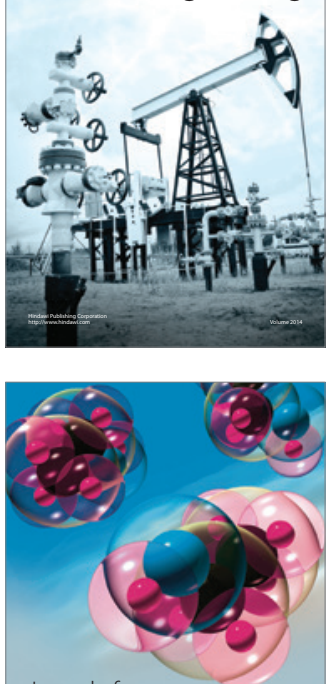

Fuels
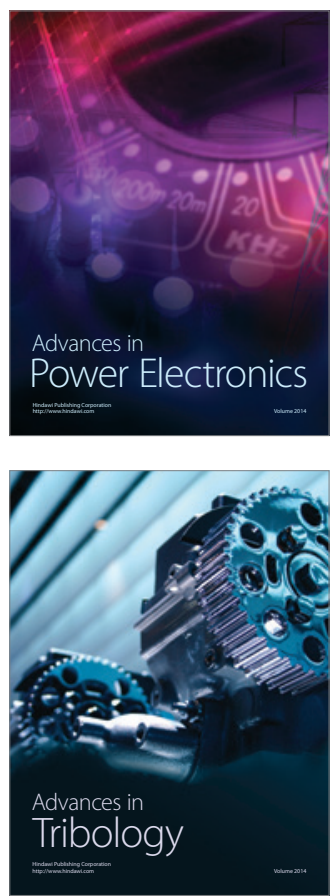

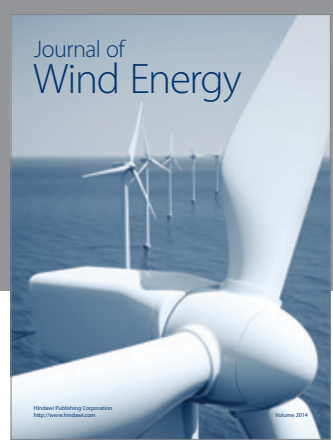

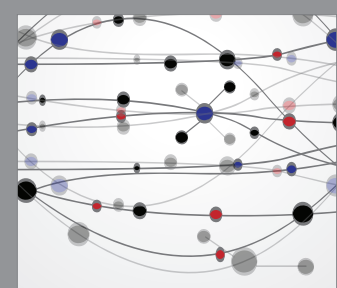

The Scientific World Journal

Submit your manuscripts at http://www.hindawi.com

Journal of

Structures
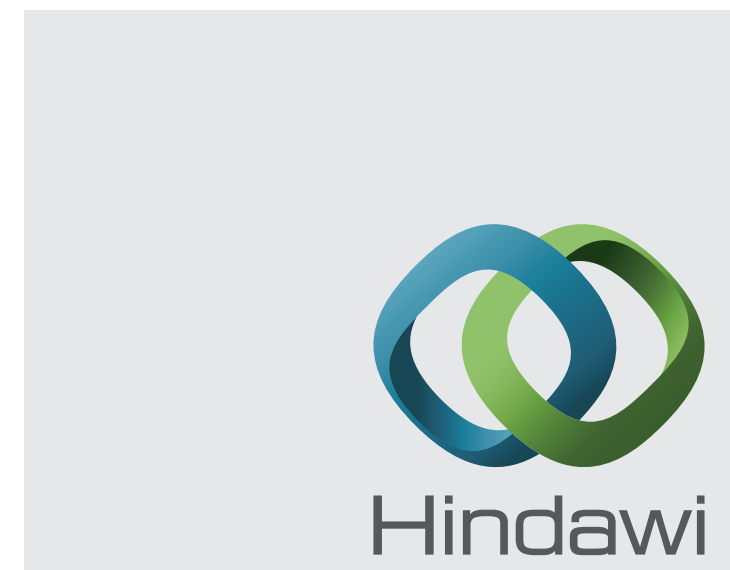

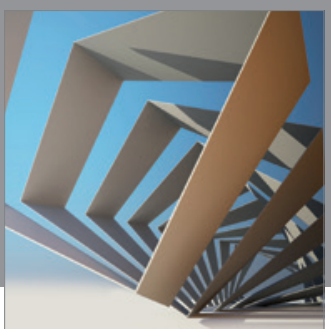

Rotating

Machinery
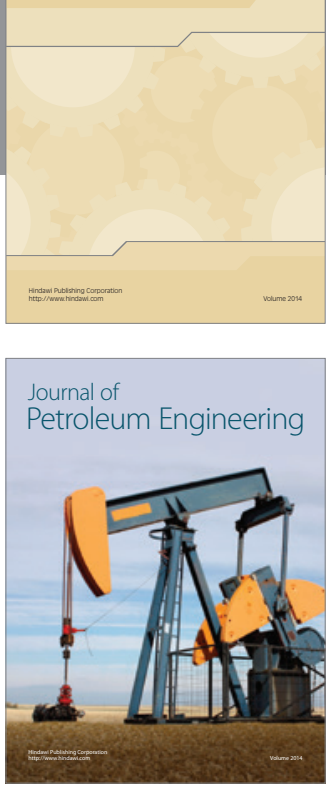

Journal of

Solar Energy
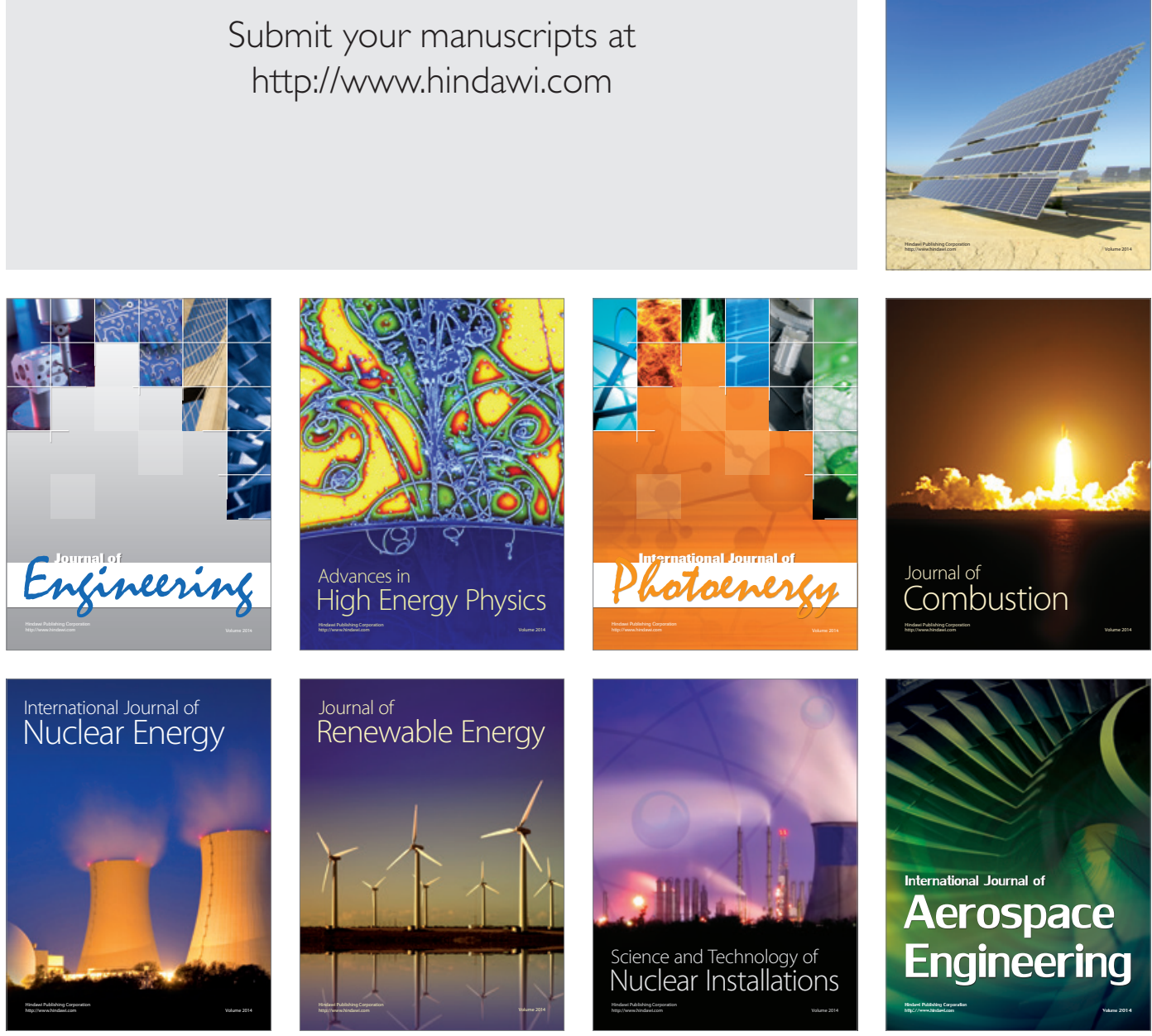